\title{
Deep Reinforcement Learning-Based Spectrum Allocation in Integrated Access and Backhaul Networks
}

\author{
Wanlu Lei ${ }^{\circledR}$, Student Member, IEEE, Yu Ye ${ }^{\circledR}$, Student Member, IEEE, \\ and Ming Xiao ${ }^{(}$, Senior Member, IEEE
}

\begin{abstract}
We develop a framework based on deep reinforcement learning (DRL) to solve the spectrum allocation problem in the emerging integrated access and backhaul (IAB) architecture with large scale deployment and dynamic environment. The available spectrum is divided into several orthogonal sub-channels, and the donor base station (DBS) and all IAB nodes have the same spectrum resource for allocation, where a DBS utilizes those sub-channels for access links of associated user equipment (UE) as well as for backhaul links of associated IAB nodes, and an IAB node can utilize all for its associated UEs. This is one of key features in which 5G differs from traditional settings where the backhaul networks are designed independently from the access networks. With the goal of maximizing the sum log-rate of all UE groups, we formulate the spectrum allocation problem into a mix-integer and non-linear programming. However, it is intractable to find an optimal solution especially when the IAB network is large and time-varying. To tackle this problem, we propose to use the latest DRL method by integrating an actor-critic spectrum allocation (ACSA) scheme and deep neural network (DNN) to achieve real-time spectrum allocation in different scenarios. The proposed methods are evaluated through numerical simulations and show promising results compared with some baseline allocation policies.
\end{abstract}

Index Terms-Integrated access and backhaul, spectrum allocation, deep reinforcement learning.

\section{INTRODUCTION}

$\mathbf{T}$ HE GLOBAL traffic data rate is estimated to continuously increase with an annual rate of $30 \%$ between 2018 to 2024 due to the exponential increase in the number of mobile broadband subscribers such as smartphones and tablets [1]. An effective solution to handle these relentless

Manuscript received December 1, 2019; revised March 15, 2020; accepted April 27, 2020. Date of publication May 6, 2020; date of current version September 9, 2020. The work of Ming Xiao is supported in part by EU Marie Sklodowska-Curie Actions Project entitled "High-Reliability Low-Latency Communications with Network Coding," and ERA-NET Smart Energy Systems SG+ 2017 Program, "SMART-MLA" with Project number 89029 (and SWEA number 42811-2), and Swedish Strategic Research Foundation Project "High-Reliable Low-Latency Industrial Wireless Communications." The associate editor coordinating the review of this article and approving it for publication was M. Guizani. (Corresponding author: Wanlu Lei.)

Wanlu Lei is with the School of Electrical Engineering and Computer Science, KTH Royal Institute of Technology, 11428 Stockholm, Sweden, and also with Ericsson AB, 16480 Stockholm, Sweden (e-mail: wllei@kth.se).

Yu Ye and Ming Xiao are with the School of Electrical Engineering and Computer Science, KTH Royal Institute of Technology, 11428 Stockholm, Sweden (e-mail: yu9@kth.se; mingx@kth.se).

Digital Object Identifier 10.1109/TCCN.2020.2992628 demands is to increase the network capacity through network densification and frequency reuse. However, the acquisition cost of site and fiber deployments is one of the biggest challenges in implementing densification at a large scale in the networks. According to [2], the fiber optic link costs about $85 \%$ of total installation and trenching. To handle this challenge, the investigation of self-backhauling, where same spectrum resources can be shared by both the access and the backhaul transmissions, has attracted much attention due to its flexibility and cost efficiency compared with fiber-based wired solutions. Recently, the emergence of IAB, which is also refereed to as self-backhauling networks in the literature, has gained much attention, and it becomes one of the key scenarios in $5 \mathrm{G}$ and future millimeter wave (mmWave) networks. Actually, 3GPP Release 15 also involved a new study item (SI) to analyze the feasibility of IAB deployment for New Radio (NR). Unlike traditional wireless backhauling, IAB nodes can use the same radio resource for access and backhaul links. Besides, the dense deployment of IAB nodes together with the aggressive frequency reuse brings about a direct consequence of challenges in providing reliable backhaul transmission and mitigating severe co-tier and cross-tier interference.

The emerging architecture of IAB has motivated lots of recent research activities from both academia and industries. References [3], [4] analyze a two-tier heterogeneous cellular networks (HetNeT) with IAB where a DBS provides mmWave backhaul to the IAB nodes, and concluded that due to the bottleneck of backhaul links between DBS and IAB nodes, it is difficult to improve user rates in an IAB settings. In [5], it evaluates the end-to-end performance of IAB architecture through system-level full-stack simulation and shows that IAB can be a viable solution to effectively relay traffic in very congested networks. The investigation in finding optimal routing and scheduling strategies has also been studied in [6]. As many prior works consider IAB in mmWave scenarios due to the advantage of large bandwidth offered by mmWave, none has considered the impact of limited backhaul capacity together with spectrum allocation problem in the sub- $6 \mathrm{Ghz}$ setup of $\mathrm{IAB}$, to the best of our knowledge.

IAB settings in sub- 6 Ghz frequency actually plays a significant role in many practical scenarios in $5 \mathrm{G}$ and beyond networks. Such as in public safety applications, where a network extension is required by adding more IAB nodes to 
reach higher coverage, or where a temporary IAB network needs to be deployed collaboratively with existing DBS in certain emergency situations. The spectrum allocation schemes in these scenarios are too divergent from the conventional setup and make it difficult to apply any off-the-shelf methods. Some tailored framework and algorithms for spectrum allocation need to be investigated to adapt to the system dynamics and fulfil real time requirements.

The spectrum allocation problem has been extensively studied in [7]-[10] and is usually solved by formulating as an optimization problem. Nevertheless, most of these methods need accurate or complete information about the network, such as channel state information (CSI) or are achieved with very expensive computational complexity. Besides, network dynamics are seldom addressed and many solutions to the optimization problem are solved in only a snapshot of the network or valid in a specific network architecture. These model-dependent schemes are indeed inappropriate for complex and highly time-varying scenarios. In an established network environment, base station (BS) employs static spectrum allocation strategy, such as full-reuse or fixed orthogonal allocation methods to ease the system computation and implementation complexities. However, ultra dense IAB environment makes full-spectrum reuse or other static schemes less efficient due to the severe co-tier interference and crosstier interference introduced by neighboring BSs, as shown in Fig. 1. The rate of UE associated with IAB is determined by the minimum rate of backhaul link and access link, which makes the final rate sensitive to the spectrum allocation strategies. When more IAB nodes are deployed and more spectrum resource becomes available in the IAB network, the solution space for spectrum allocation increases exponentially. To address this issue, we will exploit latest findings in reinforcement learning (RL) and DNN to develop a scalable and model-free framework to solve this problem. The framework is expected to have capability to effectively adapt with IAB network topology changes, large-scale sizes and different real time system requirements. We firstly consider a centralized approach in this work, and leave the distributed approach to future work.

Many exciting news in Artificial Intelligence(AI) have just happened in recent years [11]. Learning-based method in playing Atari 2600 video games outperforms all previous approaches and surpasses human expert, Alpha Go [12] beats the best human players in the game of Go. Very soon the extended algorithm AlphaGo Zero beats AlphaGo by 100-0 without supervised learning on human knowledge. The magic behind these happenings is the well-known RL algorithm. The development of Q-learning is a big breakout in the early days of RL [13]. The classical Q-learning is demonstrated to perform well in small-size models but becomes less efficient when the network is scaling up. RL combined with the state-of-theart technique DNN addresses the problem and its capability of handling large state spaces to provide a good approximation of Q-value inspires remarkable upsurge of research works in wireless communication problems. The deep Q-learning based power allocation problem has been considered in [14] and [15]. In [16], it exploits DRL and proposes an intelligent modulation and coding scheme (MCS) selection algorithm for the primary

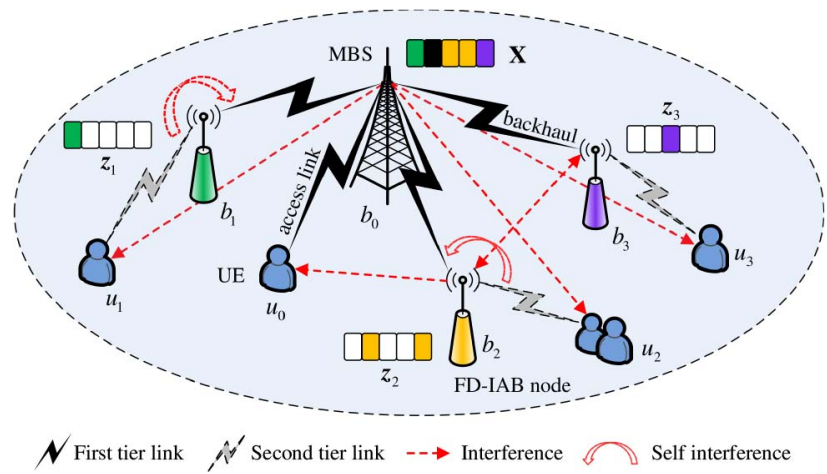

Fig. 1. Integrated Access and Backhaul With shared Spectrum Resource.

transmission. A similar approach has also been proposed in [8] for dynamic spectrum access in wireless network. However, directly applying deep Q-network (DQN) to the spectrum allocation problem is not feasible, because the action space can be very large with increasing of the network size and available spectrum, and it consumes much longer time for convergence. Recent work from DeepMind has introduced an actor-critic method that embraces DNN to guide decision making [17] for continuous control. This model can be applied to large discrete action space [18] to tasks having up to one million actions.

Based on our observations, it can be concluded that DRL is a promising technique for wireless communication systems in the future. On one hand, the large amount of data from intelligent radio can be used for training and predicting purpose, and hence improving system performance by better decision making (spectrum mapping and allocation for UE). On the other hand, it is able to handle highly dynamic time-variant system with different network setups and UE demands. Moreover, another appealing advantage in RL is the flexibility in reward function design. With proper designed reward at each step, the system performance can be improved according to different desired objective. The interaction between the agent (can be a center located controller or distributed UE) and the environment are beneficial in the learning process such that the agent can learn to select appropriate strategies to gain more rewards.

In what follows, we will study the spectrum allocation problem in the IAB architecture. To the best of our knowledge, we may be the first to investigate practical DRL-based spectrum allocation framework in an IAB setup. Our main contributions are as follows:

- We formulate the spectrum resource allocation problem in an IAB setup into a non-convex mix integer programming, the objective of which tries to maximize a generic network utility function.

- We develop a novel model-free framework based on double deep Q-network (DDQN) and actor-critic techniques for dynamically allocating spectrum resource with system requirements. The proposed architecture is simple for implementation and does not require CSI related information. The training process can be performed offline at a centralized controller, and the updating is required only when significant changes occur in IAB setup. 
- We show that with proposed learning framework, the improvement of the existing policy can be achieved with guarantee, which yields a better sum log-rate performance. We evaluate the learning speed of two algorithms and show that actor-critic based learning applying policy gradient for Q-value maximization is more effective in convergence speed than value-based DDQN when action-space is large.

- We show that the actor-critic based spectrum allocation scheme outperforms in system sum log-rate compared with the static allocation strategy when the network size increases.

The remaining of this paper is organized as follows. We first formulate the spectrum allocation problem in IAB setup in Section II. To solve the optimization problem, we propose two DRL-based algorithms in Section III. Simulation results are presented in Section IV to demonstrate the effectiveness of the proposed approaches. We conclude the paper in Section V.

\section{SYSTEM MODEL}

Consider a downlink (DL) transmission two-tier IAB network, where DBS $b_{0}$ is located at the center of the network with IAB nodes deployed uniformly within the coverage area. We denote the set of IAB nodes as $\mathcal{B}^{-}=\left\{b_{l} \mid l=\right.$ $1,2, \ldots, L\}$. Each IAB is equipped with two antennas: the receiving antenna at the mobile termination (MT) side for the wireless backhaul with DBS, and the transmitting antenna at digital unit (DU) side for access to serve its associated UE groups. IAB nodes are assumed to be full-duplex (FD) capable of certain self-cancellation ability. FD is an important topic in the IAB setup. Since our focus in the work is a general spectrum allocation framework, the research for the self-cancellation scheme is not in the scope of this work. Let $\mathcal{B}=\left\{b_{0}\right\} \cup \mathcal{B}^{-}$denote the set of all base stations (BS) including the DBS and all IAB nodes, where $|\mathcal{B}|=1+L$. The total bandwidth in which each BS can operate is divided into $M$ orthogonal sub-channels, denoted by $\mathcal{M}=\{1,2, \ldots, M\}$. We assume that each BS has a representative UE group associated to it where all UEs in this group are collocated. For notation simplicity, we use similar denotation for UEs as in BSs. The UE group associated with DBS is denoted as $u_{0}$, while the set of UE groups associated with IAB nodes is denoted as $\mathcal{U}^{-}=\left\{u_{l} \mid l=1,2, \ldots, L\right\}$, where $u_{l}$ denotes the UE group that is associated with IAB node $b_{l}$. The complete UE group set is denoted as $\mathcal{U}=\left\{u_{0}\right\} \cup \mathcal{U}^{-}$. As often described in IAB networks, the IAB nodes are treated as UEs to its associated parent, i.e., the DBS in our setup. Thus, the first-tier receivers set is denoted as $\mathcal{F}_{1}=\left\{u_{0}\right\} \cup \mathcal{B}^{-}$, while the second-tier receivers set is $\mathcal{U}^{-}$.

Note that each BS has $M$ spectrum resources for allocation, and we assume that access and backhaul links share the same pool of resource through $M$ orthogonal sub-channels. The spectrum resource of the IAB node is dedicated assigned to its associated UE group. We denote the spectrum-allocation vector at the $i$-th $\mathrm{IAB}$ as $\mathbf{z}_{i}=\left[z_{i}^{1}, \ldots, z_{i}^{M}\right]^{T}$, where $z_{i}^{m} \in\{0,1\}$, $m \in \mathcal{M}, i \in \mathcal{B}^{-}$. When the $m$-th sub-channel is used by the $i$-th IAB node, $z_{i}^{m}=1$ otherwise $z_{i}^{m}=0$. Differently, the spectrum resource at DBS can be utilized either by its associated UE group for access transmission or the corresponding associated IAB node for backhaul transmission. We denote the spectrum-allocation vector at DBS for its $f$-th receiver as $\mathbf{x}_{f}=\left[x_{f}^{1}, \ldots, x_{f}^{M}\right]^{T}$, where $x_{f}^{m} \in\{1,0\}$ and $f \in \mathcal{F}_{1}$. For example, if $x_{u_{0}}^{m}=1$, the DBS utilizes the $m$-th subchannel for access link of its associated UE $u_{0}$, and for backhaul link to the $i$-th IAB node if $x_{i}^{m}=1, i \in \mathcal{B}^{-}$. Thus, for notation simplicity, the allocation mappings is denoted as $\mathbf{X}=\left[\mathbf{x}_{1}, \ldots, \mathbf{x}_{1+L}\right]^{T}$ and $\mathbf{Z}=\left[\mathbf{z}_{1}, \ldots, \mathbf{z}_{L}\right]^{T}$ for the firsttier receivers and the second-tier receivers, respectively. We assume that DBS utilizes all its spectrum resource in the required coverage area.

Without loss of generality, we assume that several consecutive sub-carriers are grouped into one to form a spectrum sub-channel, and the channel fading is the same within one sub-channel and independent across different sub-channels. At a coherence time period, we denote the downlink channel gain from transmitter $i$ to receiver $j$ at the $m$-th sub-channel as

$$
g_{i, j}^{m}=\alpha_{i, j} h_{i, j}^{m}
$$

where $i \in \mathcal{B}, j \in \mathcal{F}_{1} \cup \mathcal{U}^{-}$. For example, if $i=b_{0}$ and $j \in \mathcal{B}^{-}, g_{i, j}^{m}$ represents the backhaul link channel gain between DBS and IAB node. And $h_{i, j}^{m}$ is the frequency dependent small-scale fading which undergoes Rayleigh fading, i.e., $h_{i, j}^{m} \sim \exp (1) . \alpha_{i, j}$ represents the large-scale fading coefficient, and it is a function of distance between $i$ and $j$ including the pathloss and shadowing effect.

During the transmission session between $i$ to $j$ at the $m$-th sub-channel, the interference to the target receiver is introduced by other BSs downlink transmissions on the same sub-channel. As DBS the transmitter in the first-tier, the received signal-to-interference-and-noise ratio (SINR) of UE $u_{0}$ and IAB node $b_{l}$ over the $m$-th sub-channel is expressed as

$$
\Gamma_{b_{0}, u_{0}}^{m}=\frac{P_{b_{0}} g_{b_{0}, u_{0}}^{m} x_{u_{0}}^{m}}{\underbrace{\sum_{i \in \mathcal{B}^{-}} g_{i, u_{0}}^{m} z_{i}^{m}}_{\text {co-tier interference }}+\sigma_{u}^{2}}
$$

and

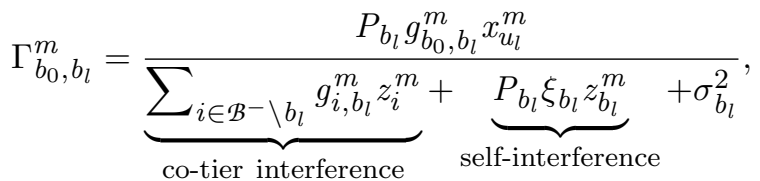

where $\xi_{b_{l}}$ is the self-interference cancellation ability of IAB node $b_{l}$, and $\sigma_{u}^{2}$ and $\sigma_{b_{l}}^{2}$ are noise power for the UE and IAB node, respectively. The received SINR for the second-tier UE groups is

$$
\Gamma_{b_{l}, u_{l}}^{m}=\underbrace{\underbrace{\sum_{b_{l}} g_{b_{l}, u_{l}}^{m} z_{l}^{m}}_{\text {cross-tier interference }} g_{i, u_{l}}^{m} z_{i}^{m}}_{\text {co-tier interference }}+\underbrace{g_{u}^{m}}_{b_{0}, u_{l} \sum_{j \in \mathcal{F}_{1}} x_{j}^{m}}+\sigma_{u}^{2} .
$$


The instantaneous rate of $u_{0}$ is determined by the spectrum allocation matrix $\mathbf{X}$ and $\mathbf{Z}$ that is expressed as

$$
C_{u_{0}}(\mathbf{X}, \mathbf{Z})=\sum_{m=1}^{M} \log \left(1+\Gamma_{b_{0}, u_{0}}^{m}(\mathbf{X}, \mathbf{Z})\right)
$$

Since the IAB node receives signal from DBS and transmits to its associated UE group, the instantaneous rate of the secondtier UE $u_{l}$ is decided by the minimum value of the backhaul rate and the access rate, which is given by

$$
C_{u_{l}}(\mathbf{X}, \mathbf{Z})=\min \left(C_{b_{0}, b_{l}}(\mathbf{X}, \mathbf{Z}), C_{b_{l}, u_{l}}(\mathbf{X}, \mathbf{Z})\right)
$$

where $C_{b_{0}, b_{l}}$ represents the backhaul link rate from DBS to IAB node $b_{l}$, and $C_{b_{l}, u_{l}}$ is the access link rate for corresponding associated UE group of the IAB node $b_{l}$.

Based on the description above, we are interested in maximizing a generic network utility function $f(x)$ as expressed below

$$
\begin{aligned}
(\mathrm{P} 1): \max _{\boldsymbol{X}, \boldsymbol{Z}} & \sum_{j \in \mathcal{U}} f\left(C_{j}(\boldsymbol{X}, \boldsymbol{Z})\right), \\
\text { s.t. } \quad & C_{j} \geq \Omega_{j}, \forall j \in \mathcal{U} ; \\
& \sum_{f \in \mathcal{F}_{1}} x_{f}^{m}=1, m \in \mathcal{M} ; \\
& \sum_{f \in \mathcal{F}_{1}} \sum_{m \in \mathcal{M}} x_{f}^{m} \leq M ; \\
& \sum_{m \in \mathcal{M}} z_{i}^{m} \leq M, \forall i \in \mathcal{B}^{-} ; \\
& x_{f}^{m}, z_{i}^{m} \in\{0,1\}, \forall f \in \mathcal{F}_{1}, \forall i \in \mathcal{B}^{-}, \forall m \in \mathcal{M},
\end{aligned}
$$

where (7b) is Quality of Service (QoS) requirement of each UE group in the system, (7c) and (7d) are constraints for allocation vector of DBS, such that each sub-channel can be only allocated to either access or backhaul transmission. (7e) and (7f) are constraints for the IAB node allocation vector that only $M$ sub-channels are available to employ. The spectrum allocation problem (P1) is non-convex mix integer programming, which has been shown to be NP-hard [19]. The solution of (P1) becomes intractable especially when the network size and available sub-channels increase.

The function $f(x)$ can be designed to obtain a certain network utility. Since we assume that each BS always has a UE group associated to it. Since each UE group requires to reach a certain rate level for transmission purpose at all time step, we consider the objective which aims at maximizing the global system rate. This is known as proportional fairness [20], and it yields $f(x)=\log (x)$.

To this end, the spectrum resource allocation problem of the IAB network is to design a spectrum allocation scheme for backhaul and access link transmission using the same resource pool. Spectrum resources expressed through binary variables $x_{f}^{m}$ and $z_{i}^{m}\left(\forall f \in \mathcal{F}_{1}, \forall i \in \mathcal{B}^{-}, \forall m \in \mathcal{M}\right)$ are designed to maximize the network utility function as described in (P1).

\section{REINFORCEMENT LEARNING IN RESOURCE ALLOCATION}

\section{A. Background of Reinforcement Learning}

The essential idea behind RL is to learn good policy through trail-and-error interaction with a dynamic environment [21], [22]. RL dates back to the early days of work in psychology, neuroscience and computer science. It receives massive attention recently in the machine learning communities. It has been widely used in decision making problems, mainly for the reason that it does not require prior knowledge of the system model and can be effectively adapted to stochastic transitions in the system. The RL algorithm is initially designed to find the optimal policy with a fully observable Markovian environment. RL methods can also be applied in various settings such as non-Markovian environment and is shown to have good performance.

Let $\mathcal{S}$ denote a set of possible states in the IAB network environment, and $\mathcal{A}$ denote a discrete set of actions. The problem can be modeled as an Markov Decision Process (MDP). The transition probability distribution is the set $\mathcal{P}: \mathcal{S} \times \mathcal{A} \times \mathcal{S} \rightarrow \mathbb{R}$, and the reward function is $r: \mathcal{S} \times \mathcal{A} \rightarrow \mathbb{R}$. Assuming discrete time steps, at each coherence time $t$, the central controller of the IAB network observes a state $\mathbf{s}_{t} \in \mathcal{S}$, and takes an action $\mathbf{a}_{t} \in \mathcal{A}$ according to a certain policy $\pi: \mathcal{S} \rightarrow \mathcal{A}$. The policy $\pi(\mathbf{a} \mid \mathbf{s})$ is the probability of taking action a conditioned on the current state $\mathbf{s}$. Therefore, the policy function must satisfy $\sum_{a \in \mathcal{A}} \pi(\mathbf{a} \mid \mathbf{s})=1$. The controller, as the agent, receives an immediate reward $r$ and the environment evolves to next state $\mathbf{s}^{\prime}$ with probability $P\left(\mathbf{s}^{\prime} \mid \mathbf{s}, \mathbf{a}\right)$. Here let $R_{t}$ be the future cumulative discounted reward at time $t$ expressed as

$$
R_{t}=\sum_{\tau=0}^{\infty} \gamma^{\tau} r_{t+\tau+1}
$$

where $\gamma \in[0,1]$ is a discounted factor for future reward. The agent is only concerned with an immediate reward if $\gamma$ is set to 0 , which is considered as myopic. As $\gamma$ approaches 1, the agent takes future reward into account more strongly. The objective of RL agent is to find a policy $\pi$ that maximizes the expected accumulative discounted reward given the stateaction pair $(\mathbf{s}, \mathbf{a})$ at time $t$. This is also defined as Q-function in the classical Q-learning algorithm

$$
Q^{\pi}(\mathbf{s}, \mathbf{a})=\mathbb{E}_{\pi}\left[R_{t} \mid \mathbf{s}_{t}=\mathbf{s}, \mathbf{a}_{t}=\mathbf{a}\right] .
$$

A commonly used off-line algorithm to find the optimal stochastic policy function $\pi^{*}(\mathbf{a} \mid \mathbf{s})$ takes greedy search, which is expressed as

$$
\pi^{*}(\mathbf{a} \mid \mathbf{s})= \begin{cases}1, & \text { if } a=\arg \max _{a \in \mathcal{A}} Q^{\pi}(\mathbf{s}, \mathbf{a}) ; \\ 0, & \text { o. } \mathrm{w} .\end{cases}
$$

The policy function is written in $\pi(\mathbf{s})$ for deterministic transitions. Further analysis on policy improvement is discussed in Section III-D. It has been proved in [23] that Q-learning can effectively converge to the optimal policy with some iterative algorithms to achieve the optimal Q-value as

$$
Q^{*}(\mathbf{s}, \mathbf{a})=r(\mathbf{s}, \mathbf{a})+\gamma \sum_{\mathbf{s} \in \mathcal{S}} P\left(\mathbf{s}^{\prime} \mid \mathbf{s}, \mathbf{a}\right) \max _{\mathbf{a}^{\prime}} Q^{*}\left(\mathbf{s}^{\prime}, \mathbf{a}^{\prime}\right) .
$$


The classical Q-learning constructs a lookup table for all state-action pairs and updates the table at each step to approach optimal Q-value. The updating of Q-value for each state-action pair usually applies the $\epsilon$-greedy policy. With probability $\epsilon$ the agent picks a random action, and with probability $1-\epsilon$ the agent takes the action that gives the maximum Q-value at the current state. This is also referred as exploration and exploitation in RL. Normally, at the beginning of the iteration, $\epsilon$ is set to 1 to enable the agent to have more chances to explore the environment with different new actions, and then it decays with the iteration episodes. After obtaining new experience through interacting with the environment by taking action a at state $\mathbf{s}$, the agent transits to next state $\mathrm{s}^{\prime}$ and updates the corresponding new Q-value

$$
\begin{aligned}
Q(\mathbf{s}, \mathbf{a}) \leftarrow & Q(\mathbf{s}, \mathbf{a}) \\
& +\beta \underbrace{\left(r(\mathbf{s}, \mathbf{a})+\gamma \max _{\mathbf{a}^{\prime}} Q\left(\mathbf{s}^{\prime}, \mathbf{a}^{\prime}\right)-Q(\mathbf{s}, \mathbf{a})\right)}_{\text {temporal difference error }},
\end{aligned}
$$

where $\beta$ is the learning rate. And $r(\mathbf{s}, \mathbf{a})+\gamma \max _{\mathbf{a}^{\prime}} Q\left(\mathbf{s}^{\prime}, \mathbf{a}^{\prime}\right)$ is the learned value for each step, the former of which is the immediate reward obtained from the environment after taking the action $\mathbf{a}$, and the latter is the estimated discounted maximum Q-value after transiting to the state $\mathbf{s}^{\prime}$ by taking action $\mathbf{a}^{\prime}$. The updating rule is called Temporal Difference (TD) method since its changes are based on the estimated error between two different times.

The above value-based Q-learning method performs well when dealing with small state and action space. However, it becomes computationally infeasible when the state space and action space are large, such as in the spectrum allocation problem at hand. This is due to that the storage of the lookup table in (12) for all state-action pairs is intolerable for largescale problems. Besides, many states are rarely visited as the state space increases, which consequently leads to much longer time to converge to the optimal Q-value. To solve these two problems, deep Q-learning is proposed to improve the classical Q-learning method by applying the DNN to approximate Q-function in lieu of the lookup table, which is called DQN.

\section{B. DQN and Spectrum Allocation}

In DQN, the Q-function in (9) is approximated by a DNN with a weight parameter $\theta$ as

$$
\tilde{Q}(\mathbf{s}, \mathbf{a} ; \theta) \approx Q(\mathbf{s}, \mathbf{a}) .
$$

The essence of DQN is to determine the weights $\theta$ for Q-function. Similar to Q-learning, at each step, the agent collects experience through interacting with the IAB network and stores this experience as the form $\left(\mathbf{s}_{i}, \mathbf{a}_{i}, r_{i}, \mathbf{s}_{i}^{\prime}\right)$ in a data set $D$. Then DQN updates its $\theta$ to minimize the loss by sampling a minibatch of size $N$ from data set $D$ as

$\theta=\arg \min _{\theta} L(\theta)=\arg \min _{\theta} \sum_{i}^{N}\left(y_{i}-\tilde{Q}\left(\mathbf{s}_{i}, \mathbf{a}_{i} ; \theta\right)\right)^{2}$,

where

$$
y_{i}=r_{i}+\gamma \max _{\mathbf{a}_{i}^{\prime}} \tilde{Q}\left(\mathbf{s}_{i}^{\prime}, \mathbf{a}_{i}^{\prime} ; \theta\right)
$$

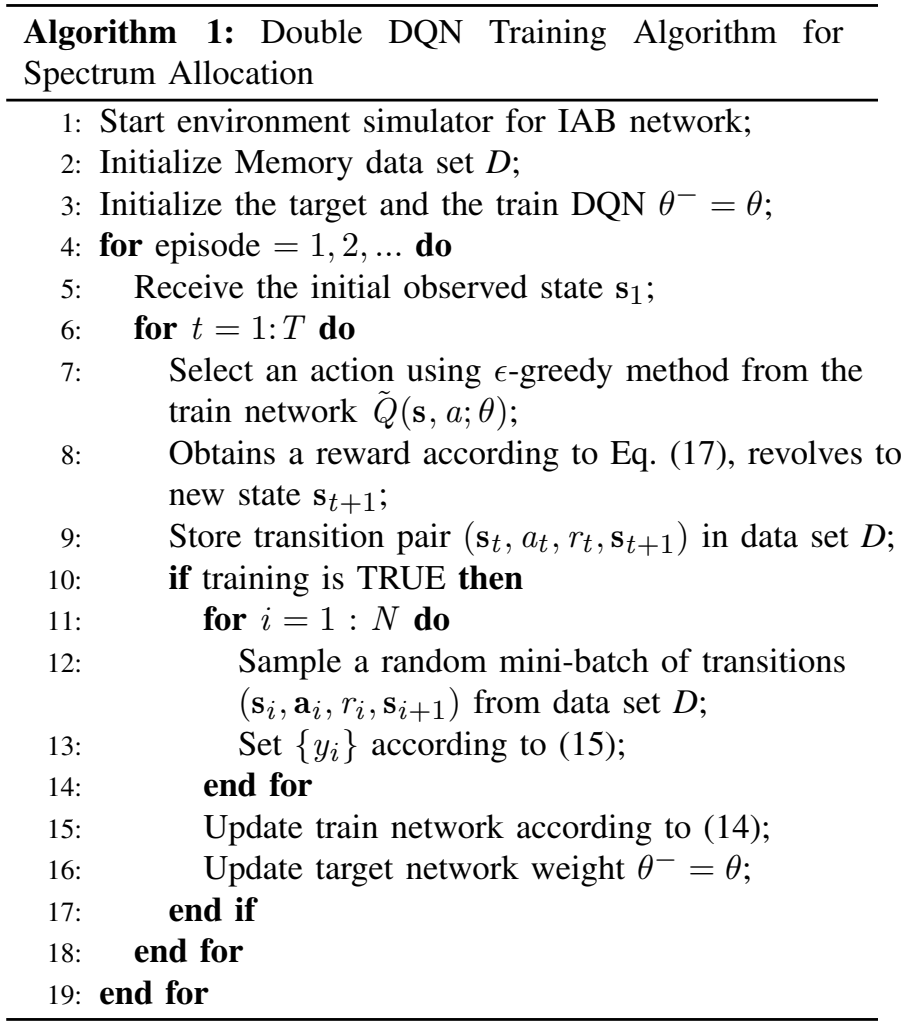

As DQN aims to greatly improve and stabilize the training procedure of Q-learning, it applies two innovative mechanisms: experience replay and periodically update target. By applying experience replay mechanism, the DQN randomly samples previous transitions in the data set $D$ to alleviate the problem of correlated data and non-stationary distributions [24]. However, the operator $\max _{\mathbf{a}^{\prime}} Q\left(\mathbf{s}^{\prime}, \mathbf{a}^{\prime} ; \theta\right)$ in (15) uses the same values as that in evaluating an action, this may lead to overoptimistic value estimates. Therefore, as the "quasi-static target network" method implies in [25], DDQN can solve this issue by introducing two DQNs: the target DQN with weights $\theta^{-}$and the train DQN with weights $\theta$ in order to remove upward bias. Parameters $\theta^{-}$are basically clones from the train network but only updated periodically according to the settings in hyperparameter. The difference of loss functions between (14) and DDQN is that the train Q-network is used to select actions while the target Q-network is used to evaluate actions. The target Q-value is calculated as

$$
y_{\mathrm{DDQN}}=r(\mathbf{s}, \mathbf{a})+\gamma \tilde{Q}(\mathbf{s}^{\prime}, \underbrace{\arg \max _{\mathbf{a}^{\prime} \in \mathcal{A}} \tilde{Q}\left(\mathbf{s}^{\prime}, \mathbf{a}^{\prime} ; \theta\right)}_{\text {selectaction } \mathbf{a}^{\prime}} ; \theta^{-}) \text {. }
$$

Finally, the stochastic gradient descent, which minimizes the loss in (14) by applying the target value in (16) is used for training over the mini-batch data set $D$ at a central controller.

At each coherence time, the agent builds its state using collected information from its associated UE and IAB nodes. To better control the transmission overhead, we restrict the state information to the quality of Service (QoS) status of all 
UEs. The center unit first computes the rate level for each UE according to (5) and (6) at $t$-th step, and then obtains the state denoted as $\mathbf{s}_{t}=\left\{s_{t, u_{0}}, s_{t, u_{1}}, \ldots, s_{t, u_{1+L}}\right\}$, where $s_{t, j} \in\{0,1\}$. And $s_{t, j}=1$ indicates that the demand of the UE group $(j \in \mathcal{U})$ has been satisfied with $C_{t, j} \geq \Omega_{t, j}$ at time $t$, and $s_{t, j}=0$ otherwise. In the simulation, we observe that the agent can learn accurately by using these QoS information without extra CSI feedback from IAB nodes or UEs. With the goal of optimizing the spectrum allocation scheme to maximize the sum log-rate, we define the action as the corresponding allocation matrix for DBS and IAB nodes as $\mathbf{a}_{t}=\left[\mathbf{X}_{t}, \mathbf{Z}_{t}\right]^{T}$. The action decision for channel allocation at IAB nodes is to evaluate the contribution of specific channels to the system objective. For example, if the condition of channel $m$ is weak at node $f \in \mathcal{B}^{-}$, and contributes more to interfering neighbors than to its associated UE signal strength, then this channel is very likely to be set to off at next state, i.e., $x_{f}^{m}=0$. The reward function is designed to optimize the network objective in (P1) for proportional fairness allocation. For all $0 \leq t \leq T$, we define the reward as

$$
r_{t}=\sum_{j \in \mathcal{U}} f\left(C_{t, j}\left(\mathbf{X}_{t}, \mathbf{Z}_{t}\right)\right) \text {. }
$$

The DQN is trained at the central controller in an off-line manner. The training procedure is stated in Algorithm 1. Both target network and train network are initialized in the same manner. We use a memory replay buffer for storing transition samples and then fetch a mini-batch of transition samples from $D$ to train the deep Q-learning networks. Though there are many investigations regarding the sampling method to improve the efficiency and accuracy of the training process [26], [27], we apply a random sampling method to take into account all possible experiences while interacting with the IAB network environment. Through step 14 in Algorithm 1, the weights $\theta^{-}$for the target network get periodically updated.

Although value-based DQN learning has been demonstrated very powerful in many application areas, there are several limitations which have been addressed in [17], [28]. For instance, as action space becomes large, the evaluation over $|\mathcal{A}|$ for action selection becomes intractable. As the spectrum allocation problem stated in (P1), the action space of the DBS is $(1+L)^{M}$, and each IAB has $2^{M}$ actions. It is obvious that the total action space increases exponentially with the number of IAB nodes and available spectrum. The required training episodes also increase significantly. Hence to improve learning efficiency, we propose a actor-critic learning method that can be more effectively adapting to the size of IAB networks.

\section{Actor-Critic Spectrum Allocation Method}

In this section we describe an Actor-Critic Spectrum Allocation (ACSA)-based framework for solving spectrum allocation problem (P1). Unlike the value-based DQN, the ACSA model is premised on having two interacting modulus. As shown in Fig. 2, it has two aptly named components: an actor and a critic. The role of the actor is to take current environment state and output an action upon certain policy, which is essentially as normal DQN does. The critic is responsible for evaluating how good the action is for the state through
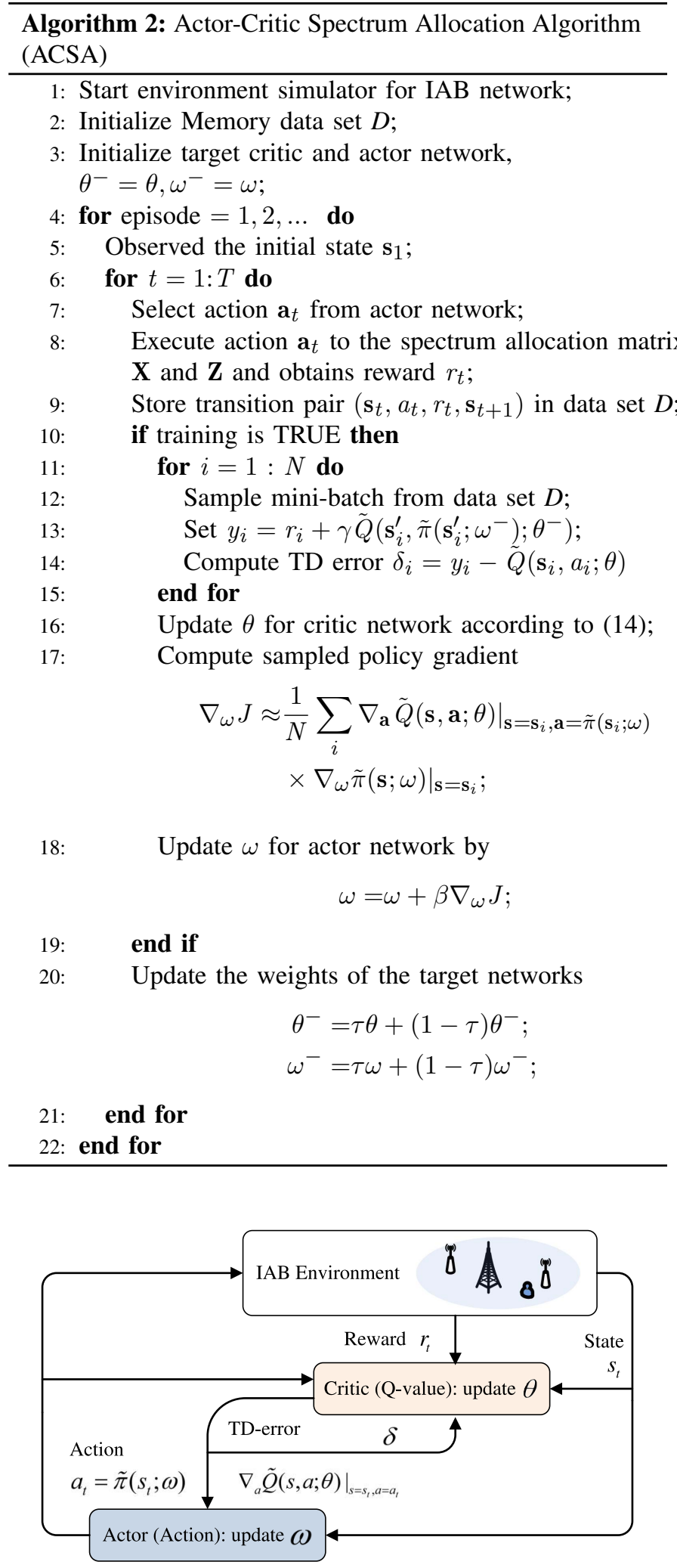

Fig. 2. Actor-Critic Spectrum allocation architecture.

returning a score from DQN by taking the action and current state as inputs. The critic is updated using TD errors similar as Q-network. The parameters of actor are updated by the returning score to improve its performance towards 
actions that bring higher score in the future. In most actorcritic models, the policy function is modeled as a probability distribution over actions $\mathcal{A}$ given the current state and thus it is stochastic. This model has been widely used given support by the policy gradient theorem [28], [29]. However, the appealing simple form for deterministic policy gradient has been demonstrated to be more efficient than the usual stochastic policy gradient in [30]. Therefore, we model the policy of the spectrum allocation problem as a deterministic decision. Following DDQN, the Q-function in (9) is approximated via DQN with a weight vector $\theta$, while the actor network uses a similar approach which approximates the policy function with the parameter $\omega$ as $\tilde{\pi}(\mathbf{s} ; \omega)$. The goal of the actor network is to determine parameter $\omega$ in policy function $\tilde{\pi}(\mathbf{s} ; \omega)$, which may result in the largest increase of Q-value. According to [30], the actor network can be updated by applying the chain rule to the expected cumulative reward $J$ with respect to the actor parameters $\omega$,

$$
\begin{aligned}
\nabla_{\omega} J & \approx \mathbb{E}\left[\left.\nabla_{\omega} \tilde{Q}(\mathbf{s}, \mathbf{a} ; \theta)\right|_{\left.\mathbf{s}=\mathbf{s}_{t}, \mathbf{a}=\tilde{\pi}\left(\mathbf{s}_{t}, \omega\right)\right]}\right. \\
& =\mathbb{E}\left[\left.\left.\nabla_{\mathbf{a}} \tilde{Q}(\mathbf{s}, \mathbf{a} ; \theta)\right|_{\mathbf{s}=\mathbf{s}_{t}, \mathbf{a}=\tilde{\pi}\left(\mathbf{s}_{t} ; \omega\right)} \nabla_{\omega} \tilde{\pi}(\mathbf{s} ; \omega)\right|_{\mathbf{s}=\mathbf{s}_{t}}\right] .
\end{aligned}
$$

We formally present the proposed actor-critic spectrum allocation method in Algorithm 2. Recall that DDQN stabilizes the learning of Q-function by experience replay and the frozen target network, i.e., the parameters of evaluating Q-network are copied to the target Q-network periodically. We similarly employ target networks for critic and actor with parameters $\theta^{-}$and $\omega^{-}$respectively. The target networks are clones of the train actor and critic networks, which are initialized in the same way as the train networks but updated with rate $\tau$ (step 18). In iteration $t$, the agent applies $\epsilon$-greedy exploration method in the actor network to obtain an action. Then the state-action pair is sent into critic train network for updating $\theta$. After receiving the gradient with respect to action output by the critic, the train network for actor updates $\omega$.

\section{Improvement of Baseline Policy}

In this subsection, we will investigate the improvement of the policy determined by ACSA, which may consequently achieve a better sum log-rate compared with baseline spectrum allocation strategy. Consider an IAB network that adopts a baseline policy $\bar{\pi}(\mathbf{s})$ which generates the state sequence $\left\{\mathbf{s}_{t}, \mathbf{s}_{t+1}, \ldots, \mathbf{s}_{T}\right\}$ from time $t$. We define the expected return under this policy $\bar{\pi}$ starting from $\mathbf{s}_{t}=\mathbf{s}$ as a value function

$$
\mathcal{V}(\mathbf{s}, \bar{\pi})=\mathbb{E}\left[R_{t} \mid \mathbf{s}_{t}=\mathbf{s}\right] .
$$

To show whether the policy $\pi^{\dagger}(\mathbf{s} ; \omega)$ obtained by ACSA is better than $\bar{\pi}$, we consider a special case where an action $\mathbf{a}^{\dagger}=$ $\pi^{\dagger}(\mathbf{s} ; \omega) \neq \bar{\pi}(\mathbf{s})$ is selected at state $\mathbf{s}$. And then the agent follows the baseline policy. We denote $Q^{\pi^{\dagger}}\left(\mathbf{s}, \pi^{\dagger}(\mathbf{s} ; \omega)\right)$ as the value obtained with this behavior. If ACSA is a better strategy, it is natural to obtain $Q^{\pi^{\dagger}}\left(\mathbf{s}, \pi^{\dagger}(\mathbf{s} ; \omega)\right) \geq \mathcal{V}(\mathbf{s}, \bar{\pi})$.

Proposition 1: If the policy $\pi^{\dagger}(\mathbf{s} ; \omega)$ of ACSA is no worse than an existing policy $\bar{\pi}$, it must obtain $\mathcal{V}(\mathbf{s}, \bar{\pi}) \leq \mathcal{V}\left(\mathbf{s}, \pi^{\dagger}\right)$.
Proof: According to (19) we derive

$$
\begin{aligned}
\mathcal{V}(\mathbf{s}, \bar{\pi}) & =\mathbb{E}\left[\sum_{\tau=0}^{\infty} \gamma^{\tau} r(\mathbf{s}, \bar{\pi}(\mathbf{s})) \mid \mathbf{s}_{t}=\mathbf{s}\right] \\
& =\mathbb{E}\left[r \left(\mathbf{s}, \bar{\pi}(\mathbf{s})+\sum_{\tau=0}^{\infty} \gamma^{\tau} r\left(\mathbf{s}_{t+1}, \bar{\pi}\left(\mathbf{s}_{t+1}\right) \mid \mathbf{s}_{t}=\mathbf{s}\right]\right.\right. \\
& =\mathbb{E}\left[r(\mathbf{s}, \bar{\pi}(\mathbf{s})) \mid \mathbf{s}_{t}=\mathbf{s}\right]+\gamma \mathbb{E}\left[R_{t+1} \mid \mathbf{s}_{t+1}=\mathbf{s}^{\prime}\right] \\
& =\mathbb{E}\left[r(\mathbf{s}, \bar{\pi}(\mathbf{s})) \mid \mathbf{s}_{t}=\mathbf{s}\right]+\gamma \mathcal{V}\left(\mathbf{s}^{\prime}, \bar{\pi}\right) .
\end{aligned}
$$

Letting $\mathbf{a}^{\dagger}=\pi^{\dagger}(\mathbf{s} ; \omega)$ be the action taken at state $\mathbf{s}_{t}$, we have

$$
\begin{aligned}
& \mathcal{V}(\mathbf{s}, \bar{\pi}) \leq Q^{\bar{\pi}}\left(\mathbf{s}, \pi^{\dagger}(\mathbf{s} ; \omega)\right) \\
&=\mathbb{E}\left[R_{t} \mid \mathbf{s}_{t}=\mathbf{s}, \mathbf{a}_{t}=\pi^{\dagger}(\mathbf{s} ; \omega)\right] \\
&\left.=\mathbb{E}_{\bar{\pi}}\left[r\left(\mathbf{s}, \pi^{\dagger}(\mathbf{s} ; \omega)\right)\right)+\gamma \mathcal{V}\left(\mathbf{s}_{t+1}, \bar{\pi}\left(\mathbf{s}_{t+1}\right)\right) \mid \mathbf{s}_{t}=\mathbf{s}\right] \\
& \leq \mathbb{E}_{\bar{\pi}}\left[r\left(\mathbf{s}, \pi^{\dagger}(\mathbf{s} ; \omega)\right)+\gamma Q^{\bar{\pi}}\left(\mathbf{s}_{t+1}, \pi^{\dagger}\left(\mathbf{s}_{t+1}\right)\right) \mid \mathbf{s}_{t}=\mathbf{s}\right] \\
&=\mathbb{E}_{\bar{\pi}}\left[r\left(\mathbf{s}, \pi^{\dagger}(\mathbf{s} ; \omega)\right)\right. \\
& \quad+\gamma \mathbb{E}\left[r\left(\mathbf{s}_{t+1}, \pi^{\dagger}\right)+\mathcal{V}\left(\mathbf{s}_{t+2}, \bar{\pi}\right) \mid \mathbf{s}_{t+1},\right. \\
&\left.\left.\quad \quad \mathbf{a}_{t+1}=\pi^{\dagger}(\mathbf{s} ; \omega)\right] \mid \mathbf{s}_{t}=\mathbf{s}\right] \\
&\left.\quad+\gamma^{2} \mathcal{V}\left(\mathbf{s}_{t+2}, \bar{\pi}\right) \mid \mathbf{s}_{t}=\mathbf{s}\right] \\
&=\mathbb{E}_{\bar{\pi}}\left[r\left(\mathbf{s}, \pi^{\dagger}(\mathbf{s} ; \omega)\right)+\gamma r\left(\mathbf{s}_{t+1}, \pi^{\dagger}\right)\right. \\
& \leq \mathbb{E}_{\bar{\pi}}\left[r\left(\mathbf{s}, \pi^{\dagger}(\mathbf{s} ; \omega)\right)+\gamma r\left(\mathbf{s}_{t+1}, \pi^{\dagger}\right)\right. \\
&\left.\quad+\gamma^{2} r\left(\mathbf{s}_{t+2}, \pi^{\dagger}\right)+\gamma^{3} \mathcal{V}\left(\mathbf{s}_{t+3}, \bar{\pi}\right) \mid \mathbf{s}_{t}=\mathbf{s}\right] \\
& \ldots \quad \mathbb{E}_{\bar{\pi}}\left[r\left(\mathbf{s}, \pi^{\dagger}(\mathbf{s} ; \omega)\right)+\gamma r\left(\mathbf{s}_{t+1}, \pi^{\dagger}\right)+\gamma^{2} r\left(\mathbf{s}_{t+2}, \pi^{\dagger}\right)\right. \\
&\left.\quad+\gamma^{3} r\left(\mathbf{s}_{t+3}, \pi^{\dagger}\right)+\cdots \mid \mathbf{s}_{t}=\mathbf{s}\right] \\
& \mathcal{V}\left(\mathbf{s}, \pi^{\dagger}\right),
\end{aligned}
$$

which completes the proof.

The proof can be extended to all states and possible actions for comparing policy of ACSA with the baseline policy. Since $\mathcal{V}\left(\mathbf{s}, \pi^{\dagger}\right)$ represents the expected sum log-rate of IAB networks, a better rate performance can be guaranteed by ACSA compared with the baseline policy $\bar{\pi}$ if Proposition 1 is satisfied.

\section{Simulation Results}

In this section, we present simulation results to demonstrate the performance of proposed methods for proportional fairness spectrum allocation in different IAB scenarios.

\section{A. Simulation Setup}

We consider an IAB network consisting of one DBS at the center, and $L$ IAB nodes which follow the Poisson point process (PPP) deployed at the radius of 250-meter from DBS. UE groups are located at the radius of 150-meter from its associated BS, the initial locations of which follow PPP. We adopt random walk model for simulating mobility of UEs in the IAB networks. The moving speed of UE $u_{j}$ at time $t$ 
TABLE I

SiMULATOR SETUP

\begin{tabular}{c|l} 
Parameter & Value \\
\hline Carrier Frequency & $2.4 \mathrm{Ghz}$ \\
Bandwidth $W$ & $20 \mathrm{Mhz}$ \\
DBS Pathloss & $34+40 \log (d)$ \\
IAB Pathloss & $37+30 \log (d)$ \\
Subchannel & Rayleigh Fading \\
Transmit power at DBS & $43 \mathrm{dBm}$ \\
Transmit power at IAB & $33 \mathrm{dBm}$ \\
Self-interference & $-70 \mathrm{~dB}$ \\
Spectral Noise & $-174 \mathrm{dBm} / \mathrm{Hz}+10 \log (W)+10 \mathrm{~dB}$
\end{tabular}

follows uniform distribution, i.e., $\nu_{j} \sim \mathcal{U}(0,2) \mathrm{m} / \mathrm{s}$, while the moving angel follows $\psi_{j} \sim \mathcal{U}(0,2 \pi)$. The distance-based path loss is characterized by the line-of-sight (LoS) model for urban environments at $2.4 \mathrm{Ghz}$, and is compliant with the LTE standard [31]. The simulator initializes an IAB network setup according to the parameters shown in Table I. We consider the proportional fairness schedule which aims to maximize $\sum_{j \in \mathcal{U}} \log \left(C_{j}\right)$. The required rate is unique among all UEs, where $\Omega_{j}=5, \forall j \in \mathcal{U}$.

We next describe the hyper-parameters used in our DDQN and ACSA methods. To better compare two algorithms, we apply the same network structure in DDQN and in critic of ACSA, which composes of three-hidden layers of fullyconnected neural networks with 500, 1000, 500 neurons. This structure is also applied to the actor network in ACSA. We found that employing different number of neurons at each hidden layer renders similar rate performance for the IAB network with small size and few sub-channels. However, the stability significantly degrades as the action space increases when few neurons are adopted. In the ACSA, the third-hidden layer is set as a customize activation function to fulfill the constraint condition in problem (P1). The softmax function is applied on each column of allocation matrix $\mathbf{X}$ for DBS since the constraint of the sum probability for allocating one typical channel equals to one. Correspondingly, this is to select the best "candidate" for each channel to get a higher score in critic networks. The sigmoid function is applied on each row of $\mathbf{Z}$ for the IAB node to select the best "combination" of sub-channels for allocation. The discount rate is $\gamma=0.9$ and the exploration rate is $\epsilon=0.9$ with a decay rate of 0.9995 . The learning rate is set to be the same for both algorithms as $\beta=10^{-4}$. The soft update rate in ACSA model is $\tau=0.01$.

The input of the proposed models is the observed state from the environment where $\mathbf{s}_{t}$. The output of DDQN is a single action for spectrum allocation. This action decision is converted to the mapping matrix $[\mathbf{X}, \mathbf{Z}]^{T}$ by a post-process. The output of the ACSA is the processed action, which can be directly applied to the allocation vectors. Thus, the output action a is with size $(2 L+1) \times M$, where the first $(L+1) \times M$ is the spectrum allocation $\mathbf{X}$ for DBS, and the next $L \times M$ is the allocation $\mathbf{Z}$ for all IAB nodes.

\section{B. Learning to Improve Fariness Schedule}

We present a typical result with $L=4$ IAB nodes, which are deployed around DBS. The available sub-channels for

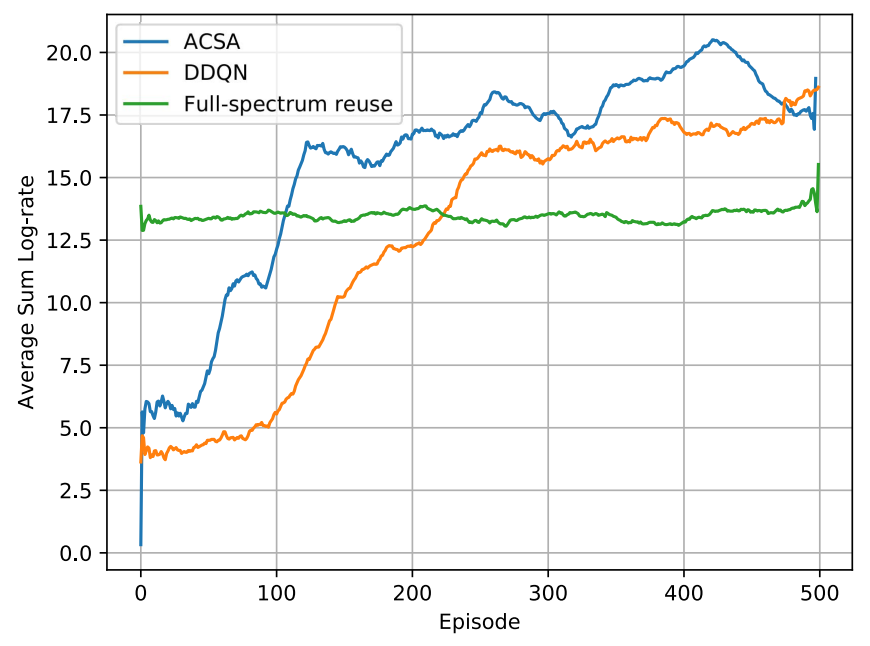

Fig. 3. Average sum log-rate for $L=4, M=10$.

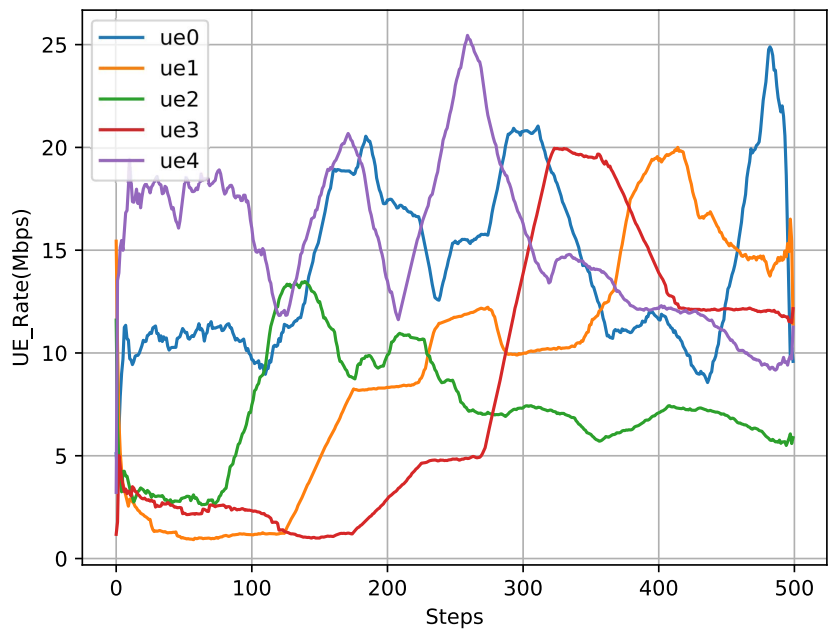

Fig. 4. Average rate of each UE applying ACSA.

resource allocation at each BS are $M=10$. As illustrated in Fig. 3, we compare the sum log-rate for the proposed DDQN and ACSA algorithms with the full-spectrum reuse strategy. The results show that both proposed methods can effectively learn from interacting with the time-varying environment and achieve better log sum-rate performance than the full-spectrum reuse strategy. It shows that reusing full-spectrum can not guarantee the optimal log sum-rate for IAB networks due to the severe interference introduced by turning on all subchannels at IAB nodes and MBS. This interference not only affects co-tier IAB nodes during backhaul transmission, but also affects the UE at DBS. In addition, the convergence speed of ACSA exceeds that of DDQN. This shows that the ACSA is more suitable for solving the problem (P1) with IAB networks. For the proposed methods, it can be seen that their performance keeps low in the beginning of learning phase. This is because the agent is exploring the environment with trial-and-error experiments. After around 120 episodes for the ACSA and 200 episodes for the DDQN, the performance of both methods intend to increase and become relatively stable. However, due to the mobility of UEs, the sum log-rate of all methods fluctuates. Unlike the static full-reuse strategy, 

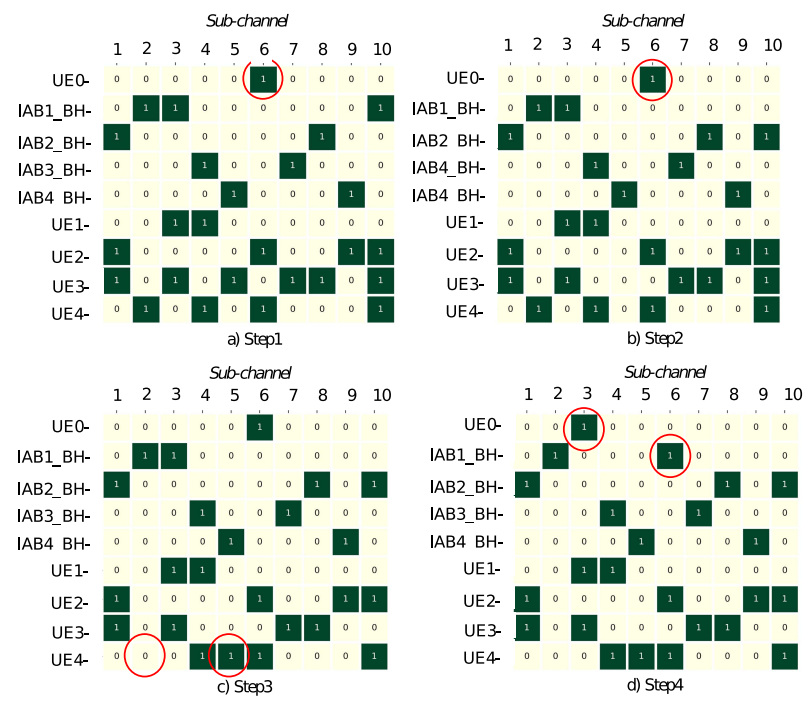

Fig. 5. Spectrum allocation in 4 snapshots.

the DRL-based methods can be trained to dynamically allocate limited spectrum resource according to the current link conditions in the IAB network, which hence achieve a better sum log-rate performance [32].

Fig. 4 illustrates one instance of the average rate of individual UE using ACSA method. The log sum-rate is relatively low in the beginning since only two UEs achieve rate requirements. This is possible since at some specific time periods, the UEs that locate close to neighbouring BSs have worse link condition than other UEs. Then the agent learns to adapt the allocation strategy to improve the log-sum rate. It can be seen that the rates of UE1, UE2 and UE3 increase rapidly after 100 steps. After around 300 steps, all UEs have achieved their system requirement. However, with increasing the rates of UE1 and UE3, the rate of UE2 is slightly decreased. This is due to that the system has reached an equilibrium allocation solution, where no UE can improve their individual rate without decreasing the rate of at least one other UE.

To better understand the learning process of DRL methods, we show how allocation decisions are made in consecutive 4 time steps in one test case, which is presented in Fig. 5. It can be seen that the 6-th sub-channel is allocated for UE0 associated with MBS at the first 3 time steps. However, at the last time-step, the 3-rd sub-channel is allocated to UE0 instead of sub-channel 6 . For all the 4 steps, UE0 experiences cross-tier interference from other two transmitting IAB nodes. As numerical result shown at this test case, as UE0 moves dynamically in the environment, the channel gain between UE0 and interfering IAB nodes $g_{i, u_{0}}, i \in \mathcal{B}^{-}$varies. From step 3 to step 4, the interference introduces by IAB node1 and node 3 decreases while by which IAB node 2 and node 4 increases. To maximize the expected cumulative sum log-rate in the system, the optimal resource mapping at step4 is to allocate sub-channel 3 to UE0 and allocate sub-channel 6 to IAB node 1 in order to guarantee the access link rate for UE0 as well as backhaul link rate for IAB node1.

Besides, we evaluate the performance gain of ACSA to a fixed policy by simulating the average sum log-rates over

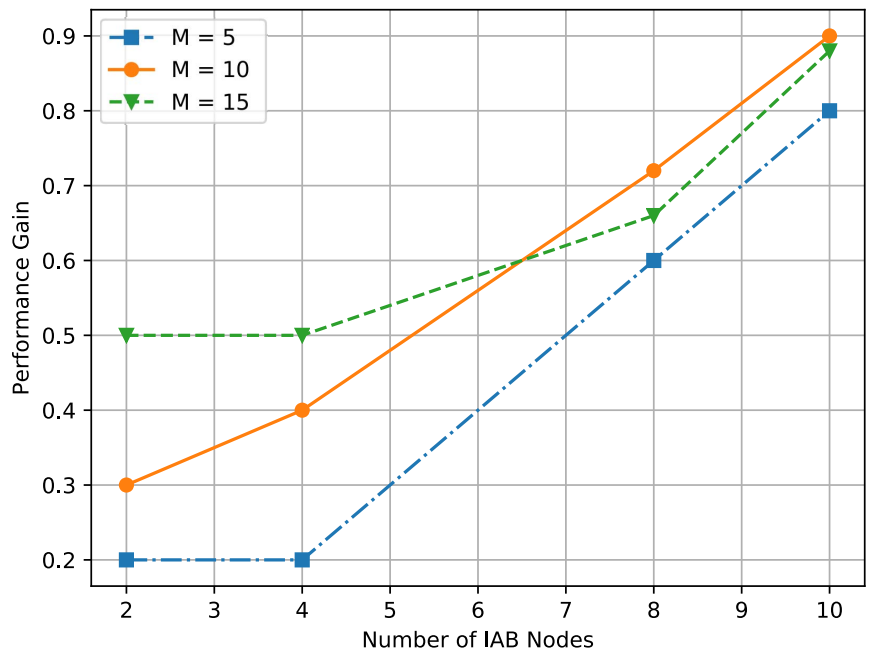

Fig. 6. Average performance gain using ACSA with different number of IAB nodes.

500 episodes with different number of IAB nodes. The performance gain is defined as Gain $=\left(\sum_{j \in \mathcal{U}} f^{\mathrm{ACSA}}\left(C_{j}\right)-\right.$ $\left.\sum_{j \in \mathcal{U}} f^{\text {fixed }}\left(C_{j}\right)\right) / \sum_{j \in \mathcal{U}} f^{\text {fixed }}\left(C_{j}\right)$. We choose 4 representative scenarios with $L=2,4,8,10$. The results in Fig. 6 show that as we increase the number of IAB nodes, the proposed DRL method can outperform the fixed allocation strategy. Since with increasing IAB nodes, the performance degradation of the static strategy dominates that of the proposed method, the performance gain increases with $L$. As more spectrum is available in the network, the proposed method shows to perform better in interference mitigation than the static allocation method.

\section{CONCLUSION}

We investigate the spectrum allocation in IAB networks by characterizing the optimization problem to maximize the sum log-rate while guaranteeing UEs system requirements. Specifically, we propose two DRL-based algorithms to solve the optimization problem at hand. We show that both algorithms can learn to adapt allocation strategy for backhaul and access transmission with dynamic network settings. Moreover, the proposed ACSA is more efficient in convergence speed compared with DDQN, and it achieves considerable performance gain in system sum log-rate comparing to static strategy especially when the size of IAB networks gets larger. Simulation results are given to show promising potentials and better sum log-rate performance of proposed methods compared with the traditional full-spectrum reuse strategy.

\section{REFERENCES}

[1] O. M. Teyeb, A. Muhammad, G. Mildh, E. Dahlman, F. Barac, and B. Makki, "Integrated access backhauled networks," 2019. [Online]. Available: http://arxiv.org/abs/1906.09298.

[2] H. A. Willebrand and B. S. Ghuman, "Fiber optics without fiber," IEEE Spectr., vol. 38, no. 8, pp. 40-45, Aug. 2001.

[3] C. Saha, M. Afshang, and H. S. Dhillon, "Bandwidth partitioning and downlink analysis in millimeter wave integrated access and backhaul for 5G," IEEE Trans. Wireless Commun., vol. 17, no. 12, pp. 8195-8210, Dec. 2018. 
[4] C. Saha, M. Afshang, and H. S. Dhillon, "Integrated mmwave access and backhaul in 5G: Bandwidth partitioning and downlink analysis," in Proc. IEEE Int. Conf. Commun. (ICC), 2018, pp. 1-6.

[5] M. Polese, M. Giordani, A. Roy, S. Goyal, D. Castor, and M. Zorzi, "End-to-end simulation of integrated access and backhaul at mmwaves," in Proc. IEEE 23rd Int. Workshop Comput. Aided Model. Design Commun. Links Netw. (CAMAD), 2018, pp. 1-7.

[6] M. Polese, M. Giordani, A. Roy, D. Castor, and M. Zorzi, "Distributed path selection strategies for integrated access and backhaul at mmwaves," in Proc. IEEE Global Commun. Conf. (GLOBECOM), 2018, pp. 1-7.

[7] V. Chandrasekhar and J. G. Andrews, "Spectrum allocation in tiered cellular networks," IEEE Trans. Commun., vol. 57, no. 10, pp. 3059-3068, Oct. 2009.

[8] B. Zhuang, D. Guo, E. Wei, and M. L. Honig, "Large-scale spectrum allocation for cellular networks via sparse optimization," IEEE Trans. Signal Process., vol. 66, no. 20, pp. 5470-5483, Oct. 2018.

[9] Z. Zhou, X. Chen, Y. Zhang, and S. Mumtaz, "Blockchain-empowered secure spectrum sharing for 5G heterogeneous networks," IEEE Netw., vol. 34, no. 1, pp. 24-31, Jan./Feb. 2020.

[10] Q. Yang et al., "Heterogeneous semi-blind interference alignment in finite-SNR networks with fairness consideration," IEEE Trans. Wireless Commun., vol. 19, no. 4, pp. 2472-2488, Apr. 2020.

[11] L. Zhang, Y.-C. Liang, and D. Niyato, "6G visions: Mobile ultrabroadband, super Internet-of-things, and artificial intelligence," China Commun., vol. 16, no. 8, pp. 1-14, 2019.

[12] D. Silver et al., "Mastering the game of go with deep neural networks and tree search," Nature, vol. 529, no. 7587, pp. 484-489, 2016.

[13] C. J. Watkins and P. Dayan, "Q-learning," Mach. Learn., vol. 8, nos. 3-4, pp. 279-292, 1992

[14] E. Ghadimi, F. D. Calabrese, G. Peters, and P. Soldati, "A reinforcement learning approach to power control and rate adaptation in cellular networks," in Proc. IEEE Int. Conf. Commun. (ICC), 2017, pp. 1-7.

[15] Y. Zhang, C. Kang, T. Ma, Y. Teng, and D. Guo, "Power allocation in multi-cell networks using deep reinforcement learning," in Proc. IEEE 88th Veh. Technol. Conf. (VTC-Fall), 2018, pp. 1-6.

[16] L. Zhang, J. Tan, Y.-C. Liang, G. Feng, and D. Niyato, "Deep reinforcement learning-based modulation and coding scheme selection in cognitive heterogeneous networks," IEEE Trans. Wireless Commun. vol. 18, no. 6, pp. 3281-3294, Jun. 2019.

[17] T. P. Lillicrap et al., "Continuous control with deep reinforcement learning," 2015. [Online]. Available: arXiv:1509.02971.

[18] G. Dulac-Arnold et al., "Deep reinforcement learning in large discrete action spaces," 2015. [Online]. Available: arXiv:1512.07679.

[19] Z.-Q. Luo and S. Zhang, "Dynamic spectrum management: Complexity and duality," IEEE J. Sel. Topics Signal Process., vol. 2, no. 1, pp. 57-73, Feb. 2008 .

[20] R. Srikant and L. Ying, Communication Networks: An Optimization Control, and Stochastic Networks Perspective. Cambridge, U.K.: Cambridge Univ. Press, 2013.

[21] R. S. Sutton and A. G. Barto, Introduction to Reinforcement Learning, vol. 2, no. 4. Cambridge, MA, USA: MIT Press, 1998.

[22] L. P. Kaelbling, M. L. Littman, and A. W. Moore, "Reinforcement learning: A survey," J. Artif. Intell. Res., vol. 4, no. 1, pp. 237-285, May 1996.

[23] J. N. Tsitsiklis, "Asynchronous stochastic approximation and Qlearning," Mach. Learn., vol. 16, no. 3, pp. 185-202, 1994.

[24] V. Mnih et al., "Playing atari with deep reinforcement learning," 2013. [Online]. Available: http://arxiv.org/abs/1312.5602.

[25] H. van Hasselt, A. Guez, and D. Silver, "Deep reinforcement learning with double Q-learning," 2015. [Online]. Available: http://arxiv.org/abs/1509.06461.

[26] T. Schaul, J. Quan, I. Antonoglou, and D. Silver, "Prioritized experience replay," 2015. [Online]. Available: arXiv:1511.05952.

[27] Z. Wang, T. Schaul, M. Hessel, H. V. Hasselt, M. Lanctot, and N. D. Freitas, "Dueling network architectures for deep reinforcement learning," 2015. [Online]. Available: arXiv:1511.06581.

[28] R. S. Sutton, D. A. McAllester, S. P. Singh, and Y. Mansour, "Policy gradient methods for reinforcement learning with function approximation," in Proc. Adv. Neural Inf. Process. Syst., 2000, pp. 1057-1063.
[29] T. Degris, P. M. Pilarski, and R. S. Sutton, "Model-free reinforcement learning with continuous action in practice," in Proc. Amer. Control Conf. (ACC), 2012, pp. 2177-2182.

[30] D. Silver, G. Lever, N. Heess, T. Degris, D. Wierstra, and M. Riedmiller, "Deterministic policy gradient algorithms," in Proc. 31st Int. Conf. Mach. Learn., 2014, pp. 387-395.

[31] E. U. T. R. Access, "Radio frequency (RF) system scenarios," Release, vol. 8, p. 56, Dec. 2011.

[32] J. Munk, J. Kober, and R. Babuška, "Learning state representation for deep actor-critic control," in Proc. IEEE 55th Conf. Decis. Control $(C D C)$, 2016, pp. 4667-4673.

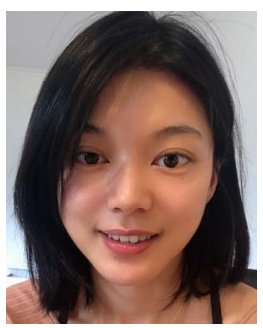

Wanlu Lei (Student Member, IEEE) received the bachelor's degree in communication engineering from the University of Electronic Science and Technology of China, Chengdu, China, in 2011, and the master's degree in communication systems from the KTH Royal Institute of Technology, Stockholm, Sweden, in 2013. From 2013 to 2017 , she worked in interconnection design with Baseband and Interconnect Department, Ericsson AB, Sweden. Since 2017, she has been working as an Industrial Ph.D. with the Division of Information Science and Engineering, School of Electrical Engineering and Computer Science, KTH Royal Institute of Technology. Her current research interests include beamforming, resource allocation, and reinforcement learning.

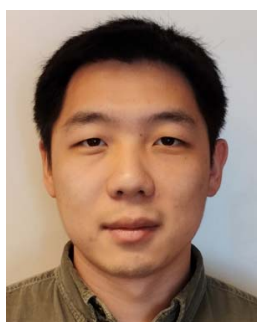

Yu Ye (Student Member, IEEE) received the bachelor's degree in communication engineering and the master's degree in communication and information systems from the University of Electronic Science and Technology of China, Chengdu, China, in 2012 and 2015, respectively. He is currently pursuing the Ph.D. degree with the Division of Information Science and Engineering, School of Electrical Engineering and Computer Science, KTH Royal Institute of Technology, Stockholm, Sweden. His current research interests include wireless caching, machine learning, and distributed optimization.

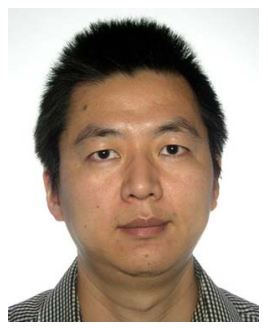

Ming Xiao (Senior Member, IEEE) received the bachelor's and master's degrees in engineering from the University of Electronic Science and Technology of China, Chengdu, in 1997 and 2002, respectively, and the Ph.D. degree from the Chalmers University of Technology, Sweden, in November 2007. From 1997 to 1999, he worked as a Network and Software Engineer with ChinaTelecom. From 2000 to 2002, he also held a position in the SiChuan communications administration. Since November 2007, he has been with the Department of Information Science and Engineering, School of Electrical Engineering and Computer Science, KTH Royal Institute of Technology, Sweden, where he is currently an Associate Professor. He was an Editor of the IEEE TRANSACTIONS ON COMMUNICATIONS from 2012 to 2017, IEEE COMMUNICATIONS LETTERS (Senior Editor since January 2015) and IEEE WIRELESS COMMUNICATIONS LETTERS from 2012 to 2016, and has been an Editor for the IEEE TRANSACTIONS ON WIRELESS COMMUNICATIONS since 2018. He was the lead Guest Editor for IEEE JSAC Special issue on "Millimeter Wave Communications for Future Mobile Networks" in 2017. He has been an Area Editor for IEEE Open Journal of the Communication Society since 2019. 